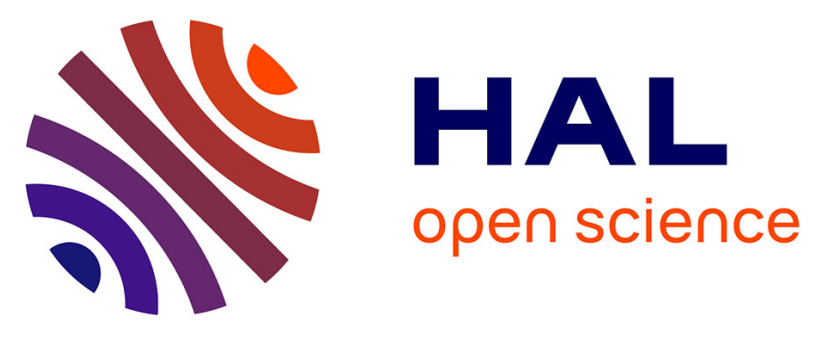

\title{
Multiscale thermomechanical approaches to SMA behaviour
}

Pierre Alart, Xavier Balandraud, André Chrysochoos, Christian Licht, Olivier Maisonneuve, Stéphane Pagano, R. Peyroux, Bertrand Wattrisse

\section{To cite this version:}

Pierre Alart, Xavier Balandraud, André Chrysochoos, Christian Licht, Olivier Maisonneuve, et al.. Multiscale thermomechanical approaches to SMA behaviour. Gérard A. Maugin; Raymonde Drouot; François Sidoroff. Continuum Thermodynamics: the art and science of modelling material behaviour, Springer Netherlands, 2002, 978-0-7923-6407-8. hal-03338458

\section{HAL Id: hal-03338458 https://hal.science/hal-03338458}

Submitted on 8 Sep 2021

HAL is a multi-disciplinary open access archive for the deposit and dissemination of scientific research documents, whether they are published or not. The documents may come from teaching and research institutions in France or abroad, or from public or private research centers.
L'archive ouverte pluridisciplinaire HAL, est destinée au dépôt et à la diffusion de documents scientifiques de niveau recherche, publiés ou non, émanant des établissements d'enseignement et de recherche français ou étrangers, des laboratoires publics ou privés. 


\title{
Multiscale thermomechanical approaches to SMA behaviour
}

\author{
$L M G C$ * \\ Laboratoire de Mécanique et Génie Civil \\ Université Montpellier II, \\ Place Eugène Bataillon, 34095 Montpellier Cedex 05, FRANCE
}

\begin{abstract}
This paper presents, in a synthetic way, several works performed on shape memory alloys (SMAs). Three scales of description are used according to whether one seeks to numerically predict the possible microstructural configurations of phases in equilibrium or the behavior of a mono- and polycrystal of SMA during a phase transition.
\end{abstract}

Keywords: SMA, phase transition, thermomechanical coupling, multiscaling.

\section{INTRODUCTION}

Shape memory alloys (SMAs) may undergo remarkable microstructural transformations: they may change the structure of their crystallographic lattice under mechanical and/or thermal loading. This transformation, called martensitic transformation, is displacive in the sense that it corresponds to a collective displacement of atoms. From a thermodynamic point of view, it belongs to the family of first order phase transitions insofar as it is accompanied by a latent heat of phase change. SMAs are being closely studied at the present time, for various reasons. From a fundamental point of view, researchers have found in the SMAs an amazing example of solid-solid phase transition, the domain of which is generally close to room temperature and thus is relatively easy to observe. From a more practical point of view, the number of industrial applications, using SMAs properties, grows day by day.

Three length scales can be introduced to study the SMA behaviour. The microscopic length scale corresponds to the crystal lattice, and is about several times the size of the unit cell; it can also be related to the representative volume element (RVE) of the continuous medium that is associated with the lattice. As for the mesoscopic and macroscopic length scales, they correspond to the RVEs of mono and polycrystal, respectively.

Since this transformation is able to generate a large variety of phase mixtures, some authors have systematically studied the possible microstructural arrangements (twinning) or rearrangement mechanisms due to straining (variants reorientation) when the material is in mechanical and thermodynamic equilibrium [Ball et al., 1987].

(*) List of authors: P. Alart, X. Balandraud, A. Chrysochoos, C. Licht, O. Maisonneuve, S. PAGANO, R. PEyrouX, B. WATTRISSE.name@lmgc.univ-montp2.fr. 
Others are interested in the phase transition itself and in its correlation with more macroscopic effects: pseudoelasiticy, self-accommodation, one-way or two-way shape memory effects, aging, etc. They are many experimental tests, mathematical modelling or numerical works on these matters. Many of these studies are directly related to engineering purposes and industrial applications, [Patoor et al., 1994].

This paper gathers and tries to synthetize the researches, performed by several teams at LMGC, which concern the thermomechanical behaviour of SMAs. In section 2 we recall the thermodynamic concepts and results used in this paper, then we will present investigations into the three scales of description. In section 3, following works of Ball and James, we show numerically computed examples of twinned microstructures. At this scale of description, the symmetries of the crystal lattice and the material frameindifference principle will be invoked to introduce non-convex potentials having multiple wells. Section 4 will be devoted to research performed at the mesoscopic scale. Two examples of "micro-meso" passage will be pointed out; they will be based on a (quasi)convexification procedure of the thermodynamic potential. The goal here will be the deduction, from microstructural considerations of section 3 , of the properties of mesoscopic (quasi-convex) potentials. A third example, more inspired by experimental results obtained on monocrystalline samples, will deal with the pseudoelastic behaviour of SMAs. We will underline the strong coupling that represents the mechanism of phase change, and the important role played by the temperature variations, particularly those induced by the latent heat. This model of monocrystal will be used, in section 5, to numerically predict the behaviour of a set of grains considered as a RVE for the polycrystal. Comparisons between calculations and experiments will be shown, considering at the same time the mechanical, thermal, and energy aspects of the phase transition.

\section{A CONVENIENT THERMOMECHANICAL FRAMEWORK}

Currently many physicists or chemists like to introduce phase transitions as unstable thermodynamic phenomena [Kondepudi et al., 1998]. The instability is in the sense defined by the Gibbs-Duhem criterion [Glansdorff et al., 1971] and is invoked in a wide range of situations [Papon et al., 1999]. Deduced from an analysis of the entropy source, the thermodynamic instability is related to a change of the convexity of the thermodynamic potentials and may consequently imply a non-monotonic mechanical behaviour. In such a context, the reversibility assumption is necessary to derive the famous equal-area rule due to Maxwell which enables us to determine the phase diagram. These ingredients have been widely exploited for describing the martensitic transformation [Müller et al., 1991], [Abeyaratne et al., 1993].

In what follows, we have chosen other ways for determining the microstructures corresponding to the possible phase mixtures at equilibrium or for introducing the phase change mechanisms in the constitutive equations. We will first assume that the phase transition is a quasi-static process possibly accompanied by irreversibilities. A convenient thermomechanical framework is the Continuum Thermodynamics [Germain, 
1973] that postulates the local state axiom. More particularly, we will use the formalism of generalised standard materials [Germain et al., 1983] for which the constitutive equations can be derived from a thermodynamic potential and a dissipation potential. The interest of modelling phase transition into such a framework is at least twofold. Theoretically, it gives a convenient mathematically consistent background to predict phase change for any thermomechanical loading. In other words, it is able to include, in the same model phase diagram, properties and kinetics of phase change induced by stress and/or temperature variations. Of course, when only stable equilibrium states of phase mixture are considered, the dissipation potential is no longer necessary and a thermostatic framework is sufficient. Experimentally, energy balance construction facilitates the interpretation of thermal and calorimetric phenomena accompanying the phase change. Besides, it leads to a better understanding of certain pseudoelastic effects such as the hysteresis loops of the stress-strain curves [Chrysochoos et al., 1993].

To describe the martensitic transformation at meso or macrolevel, the following variables are often chosen to characterise the thermodynamic state of each volume element : $T$ the absolute temperature, $\varepsilon$ a strain tensor and $x_{1}, . ., x_{\mathrm{n}}, n$ variables describing the phase mixture.

If $W$ denotes the specific Helmholtz free energy, the Clausius-Duhem inequality defines the intrinsic dissipation $d_{1}$ and the thermal dissipation $d_{2}$, both supposed to be separately positive :

$$
\left\{\begin{array}{l}
d_{1}=\sigma: \mathrm{D}-\rho W_{, \varepsilon}: \dot{\varepsilon}-\rho W_{, \mathrm{x}_{\mathrm{i}}} \dot{x}_{\mathrm{i}} \geq 0 \\
d_{2}=-\frac{q}{T} \cdot \operatorname{grad} T \geq 0
\end{array},\right.
$$

where $\sigma$ is the Cauchy stress tensor, D the Eulerian strain rate tensor, $\rho$ the mass density, and $q$ the heat influx vector. The dot stands for the material time derivative. The equality $d=d_{1}+d_{2}=0$ characterises reversible thermodynamic processes.

The intrinsic dissipation per unit volume $d_{1}$ is the difference between the inelastic energy rate $w_{i n}^{\prime}=\sigma: \mathrm{D}-\rho W_{, \varepsilon}: \dot{\varepsilon}$ and the stored energy rate $w_{S}^{\prime}=\rho W_{, \mathrm{x}_{\mathrm{i}}} \dot{x}_{\mathrm{i}}$. Deduced from both principles of thermodynamics, the local heat conduction equation is

$$
\rho C_{\varepsilon, \mathrm{x}} \dot{T}+\operatorname{div} q=d_{1}+\rho T W_{, \mathrm{T}, \varepsilon}: \dot{\varepsilon}+\rho T W_{, \mathrm{T}, \mathrm{x}_{\mathrm{i}}} \dot{x}_{\mathrm{i}}+r_{e},
$$

where $C_{\varepsilon, \mathrm{x}}$ denotes the specific heat capacity at constant $\varepsilon$ and $\left(x_{\mathrm{i}}\right)_{\mathrm{i}=1, \mathrm{n}}$, while $r_{e}$ stands for the external heat supply. The intrinsic dissipation $d_{1}$, the thermoelastic coupling term $\rho T W_{\text {,T, } \varepsilon}: \dot{\varepsilon}$ and the rate of latent heat $\rho T W_{, \mathrm{T}, \mathrm{x}_{\mathrm{i}}} \dot{x}_{\mathrm{i}}$ have been collected in the right hand member of the heat equation. Taking into account an isotropic conduction of heat $(q=-k \operatorname{grad} T)$, we underline that the left hand side becomes a partial derivative operator applied to the temperature. This property has been experimentally used to deduce the distribution of heat sources $w_{\mathrm{ch}}^{\prime}$ from temperature charts by the use of infrared techniques [Chrysochoos et al., 2000]. The volume heat source is defined by the equation :

$$
w_{\mathrm{ch}}^{\prime}=d_{1}+\rho T W_{, \mathrm{T}, \varepsilon}: \dot{\varepsilon}+\rho T W_{, \mathrm{T}, \mathrm{x}_{\mathrm{i}}} \dot{x}_{\mathrm{i}} .
$$


We emphasise that the rate of latent heat is here defined as the heat source related to the thermomechanical couplings between temperature and state variables characterising the phase mixture. An interesting property can be directly derived from the energy balance when a mechanical hysteresis loop is associated with a thermodynamic cycle of duration $C$. In [Peyroux et al., 1998], we showed that :

$$
A_{\mathrm{h}}=\oint_{\mathrm{C}} \sigma: \mathrm{D} d t=\oint_{\mathrm{C}} w_{\mathrm{ch}}^{\prime} d t
$$

where $A_{\mathrm{h}}$ is the energy associated with the hysteresis area. This result shows that the presence of a mechanical hysteresis is not only due to dissipative effects, but also to thermomechanical coupling mechanisms. The experimental investigations confirmed the main role played by the temperature on the mechanical behaviour [Chrysochoos et $a l ., 1995]$. In fact, even during quasi-static loading, the temperature variations induced by the latent heat are of the same order of magnitude as the transition domain "width". Consequently, we emphasized that these variations strongly modify the kinetics of an apparent stress-induced phase change and we claimed that the transformation is to be considered a priori as an anisothermal process. The thermomechanical couplings and the heat diffusion generate a time-dependence on SMA behaviour, to which we will return in the last sections of this paper.

\section{ATTEMPTS AT MULTISCALE MODELLING}

\subsection{Microscopic scale}

\subsubsection{A variational model for microstructures}

A neat description, which has its origins in the work of J. L. Ericksen, can be found in [Ball et al., 1987], [Ball et al., 1992], [James et al., 1989] and roughly summarized as follows. Under the Cauchy and Born rule [Ericksen, 1984], an abstract continuous medium is associated with the crystalline lattice. This medium is assumed to be thermoelastic. In the framework of the finite transformations, the free energy density function $W$ depends on the temperature $T$ and on the gradient of deformation $F$. This density function inherits some properties of a crystalline lattice encountering phase transitions:

i) $W$ is frame indifferent:

$W(Q F, T)=W(F, T), \forall(Q, F, T) \in O_{3}^{+} \times M_{3}^{+} \times R^{+}$,

$\mathrm{O}_{3}^{+}$is the set of all rotations, $\mathrm{M}_{3}^{+}$the set of all square matrices of order 3 with positive determinant,

ii) $W$ has a finite number of potential wells:

at each $T$, there exists a finite number $m(T)$ of symmetric, positive definite matrices of order 3, $U_{i}(T), i=1, m$, such that the minimizers of $W(., T)$ are the orbits $O_{3}^{+} U_{i}(T), i=1, m$. For instance « at each temperature greater than $T_{\mathrm{c}}$, the minimizers of 
$W(\cdot, T)$ are $O_{3}^{+} U_{A}(T) »$ corresponds to the existence of the austenite state at a temperature greater than the transition temperature $T_{\mathrm{c}}$. While « at each temperature lower than $T_{\mathrm{c}}$, the minimizers of $W(\cdot, T)$ are $O_{3}^{+} U_{M \mathrm{i}}(T), i=1, k »$ corresponds to the existence, at each temperature below $T_{\mathrm{c}}$, of the martensite state which appears in $\mathrm{k}$ variants, $\mathrm{k}$ being the index of the symmetry group of the martensite lattice in the symmetry group of the austenite lattice. Of course, the minimizers of $W\left(\cdot, T_{\mathrm{c}}\right)$ are the k+1 orbits $O_{3}^{+} U_{A}(T), O_{3}^{+} U_{M \mathrm{i}}(T), i=1, k$.

Finding stable equilibrium configurations of a piece of monocrystal at a uniform temperature $T$, with for instance a displacement boundary condition on its whole boundary, leads to the determination of the exact minimizers of the total energy

$$
I(\varphi)=\int_{\Omega} W(\nabla \varphi(x)) d x
$$

on the reference configuration $\bar{\Omega}$ for all admissible deformations $\varphi$. Here, the temperature acts as a parameter, thus the variable $T$ is, from now on, omitted in this section. In many situations, because of the numerous potential wells, the previous problem has no solutions. The (weak) limits of the minimizing sequences of $I$ do not minimize $I$, and they have to develop finer and finer oscillations. The fact that these spatial oscillations correspond to the observed microstructures is the basic assumption of this theory.

Our own contribution is confined to the determination of microstructures by numerical energy minimization. The aim of our numerical experiments is to check if minimizers of the discrete problems, which always exist, account for the microstructures. We denote by $Y$ the microscopic domain of observation, and $F$ the mesoscopic gradient of deformation (i. e. the average on $Y$ of the microscopic gradient of deformation).

\subsubsection{Numerical experiments at microscale}

In contrast with most previous computations [Collins, 1993] we do not use, in this part, either finite elements or gradient-like algorithms, but a method using a trigonometric interpolation and a decoupling of the deformation fields from their gradients. Saddle points of an augmented Lagrangian:

$$
L(\varphi, q ; \lambda)=\int_{\Omega}\left\{W(q)+\lambda:(\nabla \varphi-q)+\frac{r}{2}|\nabla \varphi-q|^{2}\right\} d x, \quad r>0
$$

are obtained by an Uzawa algorithm:

$$
L\left(\varphi^{n+1}, q^{n+1} ; \lambda^{n}\right) \leq L\left(\varphi, q ; \lambda^{n}\right) \text { and } \lambda^{n+1}=\lambda^{n}+r\left(\nabla \varphi^{n+1}-q^{n+1}\right) .
$$

This method is worthwhile, since a step of a relaxation procedure to minimize $L\left(., ; \lambda^{n}\right)$ involves a global linear problem of Laplace type, and local nonlinear problems of minimization of a quadratic perturbation of $W$ in a suitable set of matrices. Moreover, to compute the microstructures, we take advantage of being free to choose the domain of 
computation and, to a certain extent, the boundary conditions. With $Y$ cubic and a boundary condition of place, we can use a trigonometric interpolation on a grid of order $\mathrm{N}$, rather than finite elements. This method avoids storing a stiffness matrix and the fast Fourier transform algorithm (FFT) is faster than solving the linear system involved by finite elements.

For our numerical two-dimensional experiments [Licht, 97] we have chosen an Ericksen-like energy density function [Collins, 93]:

$$
\begin{aligned}
& W(F)=\widetilde{W}(C), C=F^{T} F \\
& \widetilde{W}(C)=k_{1}\left\lfloor\operatorname{tr} C-2-\delta^{2}\right\rfloor^{2}+k_{2}\left\lfloor C_{12}^{2}-\delta^{2}\right\rfloor^{2}+k_{3}\left\lfloor C_{11}-C_{22}-\delta^{2}\right\rfloor^{2}, k_{1}, k_{2}, k_{3}, \delta>0 .
\end{aligned}
$$

If $F=x S^{+}+(1-x) S^{-}, 0<x<1, S^{ \pm}=I \pm \delta e_{2} \otimes e_{1}$, the microstructure is unique [Ball et al., 92] and is a single twinned laminate orthogonal to $e_{1}$ with phases corresponding to the wells $S^{+}, S^{-}$in proportions $x, 1-x$. Thus a valuable test of this numerical method is to check if minimizers $\varphi_{N}$ of the discretized problem account for this microstructure.

Figures 1a (resp.1b) displays $\psi\left(\nabla \varphi_{N}\right)$ for the case $x=1 / 3$ and $N=16$ (resp. $N=32$ ) where, as proposed by [Collins, 93],

$$
\psi(F)=\left|F^{T} F-\left(U^{+}\right)^{2}\right| /\left(\left|F^{T} F-\left(U^{+}\right)^{2}\right|+\left|F^{T} F-\left(U^{-}\right)^{2}\right|\right) .
$$

Except of course in the vicinity of $\partial Y$, where $\varphi_{N}$ must satisfy the boundary condition $\varphi_{N}(y)=F y, \nabla \varphi_{N}$ essentially takes the values $S^{+}$and $S^{-}$in vertical layers in proportions $x$ and $(1-x)$. Whereas $\varphi_{N}$ depends strongly on $N$, the distribution of the values of $\nabla \varphi_{N}$ does not.

Thus, our numerical method gives the microstructure on the scale of the grid, but these nice results were obtained through initial fields with oscillations of the same wavelength as the expected microstructure. Random initialization (Fig.1c) gives a less regular distribution of $S^{+}$and $S^{-}$, but the horizontal proportions are still about $x$ and $(1-x)$. Even with slowly decreasing random perturbation of the updating of the multiplier $\lambda$, we did not succeed in avoiding this kind of local minimum (Fig.1d), however the average proportions of the vertical layers are $x$ and $(1-x)$. Figures $1 \mathrm{e}-1 \mathrm{~h}$ concern the case in which $x=1 / 2$ and $S^{ \pm}$are replaced by $S^{ \pm} R, R \in O_{3}^{+}$. When $R^{T} e_{1}$ is compatible with the grid, twinning is once more obtained.

Nevertheless trigonometric interpolation seems more flexible than piecewise affine finite elements for capturing slanting oscillations, because of the Hadamard jump condition. But as in [Collins, 93], we never succeeded in trapping laminates of order two (layers within layers)! 


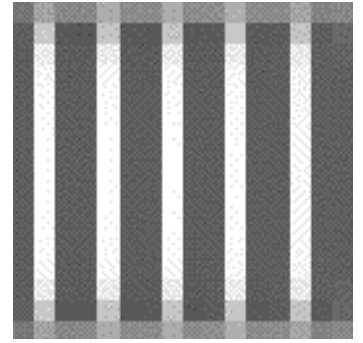

$\mathrm{a}$

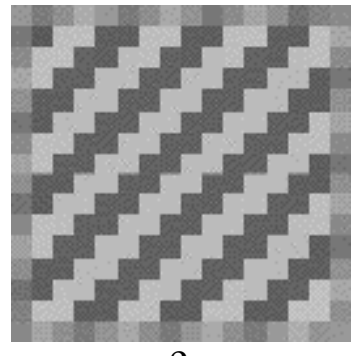

e

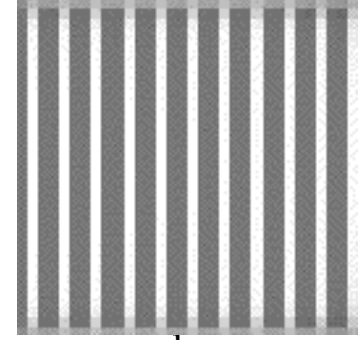

$\mathrm{b}$

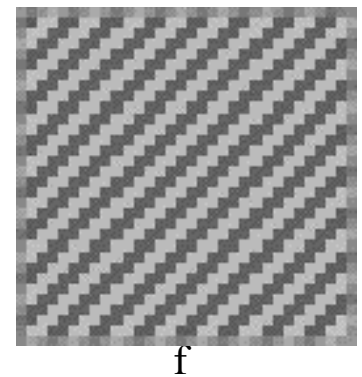

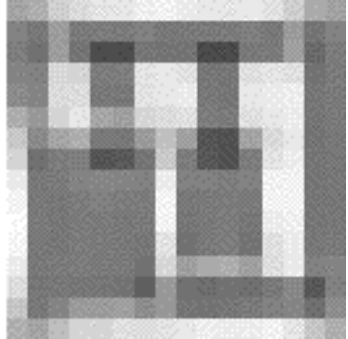

$\mathrm{C}$

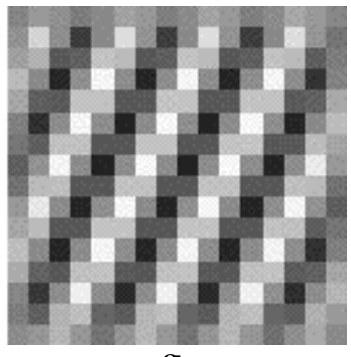

$\mathrm{g}$

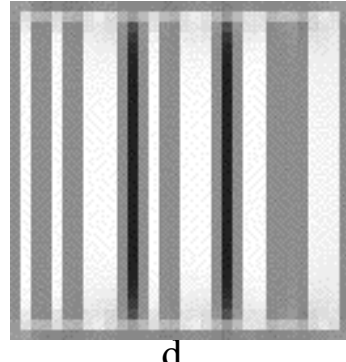

d

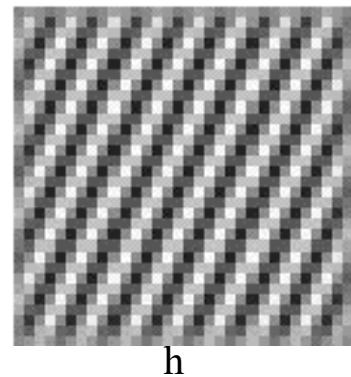

Figure 1 ; Examples of microstructures

Similar results may be obtained with a more rudimentary model in the context of small perturbations. Frémond's model may be related to the Khachaturyan-Roytburd-Shalatov theory; Kohn [Kohn, 1991] showed that this last theory is the geometrically linear analogue of the one developed by Ball and James in the context of finite deformations [Ball et al.,1992]. The free energy density function is then a function of the small strain tensor $\varepsilon$. The frequency of the oscillations of the layered microstructure depends on the mesh. Due to the choice of the residual stress tensor (introduced below) $\alpha \tau$ parallel to $I$, the orientation of the microstructure is arbitrary [Pagano, 1998].

\subsection{Mesoscopic scale}

\subsubsection{Quasiconvexification and scale transition}

If the material is assumed to be non dissipative, a first proposal for the mesoscopic strain energy density function is the quasiconvexification of the microscopic density function. The justification for this rests on the mechanical interpretation of two mathematical properties of the minimizing sequences of the total energy that were invoked in section 3.1.1, [Dacarogna, 82]. First their (weak) limits describe the average, say mesoscopic, state of a grain due to the convergence of the gradient averages in every subdomain. Next, these limits do not minimize the true total energy, but a total energy whose density is the quasiconvexification $Q W$ of the true density $W$ :

$$
Q W(F)=\operatorname{Inf}\left\{\frac{1}{|Y|} \int_{Y} W(F+\nabla u(y)) d y, u=0 \text { on } \partial Y\right\},
$$

$Q W(F)$ is the infimum of the average of the strain energy for deformations whose average gradient is $F$. Thus the mesoscopic behavior of the monocrystal is not described 
by the true (microscopic) energy density function $W$ but by an apparent density $Q W$. The main difficulty with this approach is the computation of $Q W$.

Another approach is to propose a strain energy density $W$ through phenomenological considerations and to check if it is quasiconvex (i. e. $W=Q W$ !).

\subsubsection{An academic two-phase phenomenological modeling}

Many phenomenological models of two-phase shape memory material incorporate a socalled interaction term $W^{I}=h x(1-x)$ in the free energy; $x$ is a phase proportion and $h$ a non-negative function of the temperature. Thus, if the material is assumed to be non dissipative, the strain energy is not a convex function of the linearized strain $\varepsilon$. This lack of convexity generates controversies which may lead to a rejection of the previous structure of $W^{I}$; we showed [Licht, 2000] that if $h$ is not too large the previous structure provides a good model.

We confine our attention to the case of two linearly thermoelastic phases with different stress-free strains $a_{i}$ but with the same elastic stiffness $A$; the energy density function of each phase is

$$
W^{i}(\varepsilon, T)=\frac{1}{2} A\left(\varepsilon-a_{i}(T)\right):\left(\varepsilon-a_{i}(T)\right)+w_{i}(T),
$$

and a common proposal for a family of free energies and strain energies of the mixture is

$$
\begin{aligned}
& W_{h}(\varepsilon, x, T)=\frac{1}{2} A\left(\varepsilon-a_{x}\right):\left(\varepsilon-a_{x}\right)+w_{x}+h x(1-x), \\
& \bar{W}_{h}(\varepsilon, T)=\operatorname{Min}\left\{W_{h}(\varepsilon, x, T), 0 \leq x \leq 1\right\},
\end{aligned}
$$

$a_{x}\left(\right.$ resp. $\left.w_{x}\right)$ is the convex combination in proportions $x$ and $(1-x)$ of $a_{i}\left(\operatorname{resp} . w_{i}\right)$.

Let $H=\operatorname{Min}\left\{\frac{1}{2} A(a-z):(a-z), z=k \otimes_{s} v, k, v \in R^{3}\right\}, a=a_{2}-a_{1}$.

Through Fourier analysis as in [Kohn, 91], it can be shown that

$$
\begin{aligned}
& \bar{W}_{h}(\varepsilon, T) \text { is convex if and only if } h \leq 0, \\
& \bar{W}_{h}(\varepsilon, T) \text { is not convex but quasiconvex if and only if } 0<h \leq H, \\
& \bar{W}_{h}(\varepsilon, T) \text { is not quasiconvex if and only if } H<h .
\end{aligned}
$$

Of course the second case does not occur if $\mathrm{H}=0$, that is to say, if the stress-free strains are compatible. Thus the good range for $\mathrm{h}$ ( $\underline{W}_{h}$ quasiconvex or convex) depends strongly on the compatibility of the $a_{i}$. On the other hand, incorporating a term like $W^{i}$ in a more rational definition of the macroscopic energy of a mixture like

$$
\operatorname{Inf}\left\{\begin{array}{l}
\frac{1}{|Y|} \int_{Y}\left\{\chi(y) W^{1}(e(u(y)))+(1-\chi(y)) W^{2}(e(u(y)))\right\} d y, \\
\chi \text { characteristic function, } \int_{Y} \chi(y) d y=x|Y| \text { and } u(y)=\varepsilon y \text { on } \partial Y ; e=\nabla_{s} u
\end{array}\right\},
$$


yields a free energy equal to $W_{H+h}$. Thus quasiconvexity of $\underline{W}_{H+h}$ implies $h \leq 0$ which agrees with a proposal by [James et al., 1993 ] for mismatch energy.

Moreover, if $0<h \leq H$, the function $\underline{W}_{h}(\cdot, T)-\Sigma:$. has a double-well structure for suitable $\Sigma$, so that the total mechanical energy of a body subjected to a uniform surface loading may have two strong local minimizers. Thus in some displacement-controlled tests, a negative slope of the stress-strain response curve of a specimen can be observed, while in some load-controlled test a kind of hysteresis occurs.

We also proved that approximations of the previous local minimizers can be obtained by classical finite element methods.

\subsubsection{C. algorithm: a convenient numerical tool}

The previous approach may be extended to a three phase mixture, as is usual for some models of SMAs with an austenitic phase (proportion $x_{0}$ ) and two variants of martensite (proportions $x_{-}$and $x_{+}$). We assume that the stress-free strain of the austenite is zero $\left(a_{0}=0\right)$ and the stress-free strains of the martensites are opposite $\left(a_{-}=-a\right.$ and $\left.a_{+}=a\right)$. Similarly the term $w_{-}(T)$ is equal to $w_{+}(T)$ and we define $c(T)=w_{+}(T)-w_{0}(T)$. To relate this formulation with others ([Frémond, 1987, Raniecki et al., 1992]), it is useful to introduce a residual stress tensor $\tau$ and to express the stress-free strain with respect to it, to the elastic tensor $A$ and a scalar function $\alpha(T)$ of the temperature: $a=\alpha A^{-1} \tau$. We have then 3 interaction terms between 2 phases, $h_{0-}, h_{0+}$ and $h_{-+}$. But it seems natural enough to suppose that $h_{0-}=h_{0+}=h_{0}$. By choosing $h_{+}=4 h_{0}=2 G$ [Leclercq et al., 1996, Pagano et al., 1999], the previous analysis about a two-phase mixture is preserved and the convexity and quasiconvexity properties may be recovered with respect to the single parameter $G$. Consequently, a family of free energies of the mixture may be expressed as follows:

$$
W_{G}(\varepsilon, x, T)=\frac{1}{2} A\left(\varepsilon-a_{x}\right):\left(\varepsilon-a_{x}\right)+w_{x}+h\left(x_{+}-x_{-}\right)^{2} \text {, }
$$

where

$$
a_{x}=a_{x}(T)=\left(x_{+}-x_{-}\right) \alpha A^{-1} \tau, h=h(T)=\frac{1}{2}\left(G(T)-\alpha(T)^{2} \tau: A^{-1} \tau\right) .
$$

Similarly to the 2-phase mixture, we can then distinguish 2 important values $G_{c}$ and $G_{q}$ which are determined according to our assumptions. For a bidimensional modeling, the isotropic elastic tensor ( $\lambda$ and $\mu$ the Lamé coefficients) and $\tau=\operatorname{diag}\left(\tau_{1}, \tau_{2}\right)$ we have,

$$
\begin{aligned}
& G_{c}=\frac{-\alpha^{2}}{4 \mu(\lambda+\mu)}\left[(\lambda+\mu)\left(\tau_{1}^{2}+\tau_{2}^{2}\right)-2 \lambda \tau_{1} \tau_{2}\right] \text { and } \\
& G_{q}= \begin{cases}G_{c} & \text { if }(\lambda+2 \mu) \tau_{1}-\lambda \tau_{2}<0 \text { and } \lambda \tau_{1}-(\lambda+2 \mu) \tau_{2}<0 \\
\frac{-\alpha^{2}}{\lambda+\mu} \max \left(\tau_{1}^{2}, \tau_{2}^{2}\right) & \text { otherwise }\end{cases}
\end{aligned}
$$


by convexification and quasiconvexification of the bulk energy proposed by Frémond, which corresponds to $G$ equal to zero [Frémond, 1987]. By neglecting dissipative effects, we define the bulk energy density as the marginal function of the free energy for all admissible volume proportions $\left(x=\left(x_{+}, x_{-}\right)\right.$belongs to the simplex $C$, $C=\left\{0 \leq x^{ \pm}, x^{+}+x^{-} \leq 1\right\}: M W_{G}(\varepsilon, T)=\min \left\{W_{G}(\varepsilon, x, T) ; x \in C\right\}$.

This bulk energy density is then a three-well or a two-well potential with five or three regimes described below. For an efficient numerical treatment it is useful to split it into the difference between two convex functions [Pagano et al., 1998]; the first is quadratic, represents the bulk energy density of the austenitic phase and does not depend on $G$ :

$M W_{G}(\varepsilon, T)=W_{a}(\varepsilon, T)-W_{b}(\varepsilon)$ with $W_{a}(\varepsilon, T)=W_{0}(\varepsilon, T)=\frac{1}{2} \varepsilon: A \varepsilon+w_{0}(T)$.

If $c>\frac{G}{2}$, the second convex function presents five regimes:

$$
W_{b}(\varepsilon)=\left\{\begin{array}{ll}
-\alpha \tau: \varepsilon-c & \text { if } \alpha \tau: \varepsilon \leq-c-\frac{G}{2} \\
\frac{1}{2 G}\left(\alpha \tau: \varepsilon+c-\frac{G}{2}\right)^{2} & \text { if }-c-\frac{G}{2} \leq \alpha \tau: \varepsilon \leq-c+\frac{G}{2} \\
0 & \text { if } \alpha|\tau: \varepsilon| \leq c-\frac{G}{2} \\
\frac{1}{2 G}\left(\alpha \tau: \varepsilon-c+\frac{G}{2}\right)^{2} & \text { if } c-\frac{G}{2} \leq \alpha \tau: \varepsilon \leq c+\frac{G}{2} \\
\alpha \tau: \varepsilon-c & \text { if } \alpha \tau: \varepsilon \geq c+\frac{G}{2}
\end{array} .\right.
$$

If $c \leq \frac{G}{2}$, we recover a two-well potential with three regimes:

$$
W_{b}(\varepsilon)= \begin{cases}-\alpha \tau: \varepsilon-c & \text { if } \alpha \tau: \varepsilon \leq-G \\ \frac{1}{2 G}(\alpha \tau: \varepsilon)^{2}+c+\frac{G}{2} & \text { if } \alpha|\tau: \varepsilon| \leq G . \\ \alpha \tau: \varepsilon-c & \text { if } \alpha \tau: \varepsilon \geq G\end{cases}
$$

To reproduce local instabilities inherent to phase transition [James, 1987, Zhong et al., 1996], we search for only a local minimum of the total energy $\Pi$ on a domain $\Omega$ which may still be split into two convex parts, including the bulk energy and the work of the external loading $l(v)$ :

$$
\begin{aligned}
& \Pi(v)=\phi_{1}(v)-\left(\phi_{2} \circ \varepsilon\right)(v), \\
& \phi_{1}(v)=\int_{\Omega} W_{a}(\varepsilon) d x-l(v) \text { and } \phi_{2}(\varepsilon)=\int_{\Omega} W_{b}(\varepsilon) d x .
\end{aligned}
$$

This decomposition leads us quite naturally to introduce a type II Lagrangian according to the terminology of Auchmuty [Auchmuty, 1989], depending on the Fenchel conjugate function of $\phi$, and associated with a min-min problem:

$$
L_{I I}\left(v, \tau_{2}\right)=\phi_{1}(v)+\phi_{2}^{*}\left(\tau_{2}\right)-\int_{\Omega} \tau_{2}: \varepsilon(v) d x
$$

The D.C. algorithm [Stavroulakis et al.,1993] consists in constructing a minimizing sequence of $L_{I I}$ descending, alternately, in the primal variable and the dual one. 
D.C. algorithm: $\left(u^{0}, \sigma_{2}^{0}\right)$ given, with $\left(u^{n-1}, \sigma_{2}^{n-1}\right)$ known, determine successively $u^{n}, \sigma_{2}^{n}$ as follows :

$$
\begin{array}{ll}
\text { Step 1: } & L_{I I}\left(u^{n-1}, \sigma_{2}^{n-1}\right) \leq L_{I I}\left(v, \sigma_{2}^{n-1}\right), \forall v \in U_{\mathrm{ad}}, \\
\text { Step 2: } & L_{I I}\left(u^{n}, \sigma_{2}^{n}\right) \leq L_{I I}\left(u^{n}, \tau_{2}\right), \forall \tau_{2} \in U_{\mathrm{ad}} .
\end{array}
$$

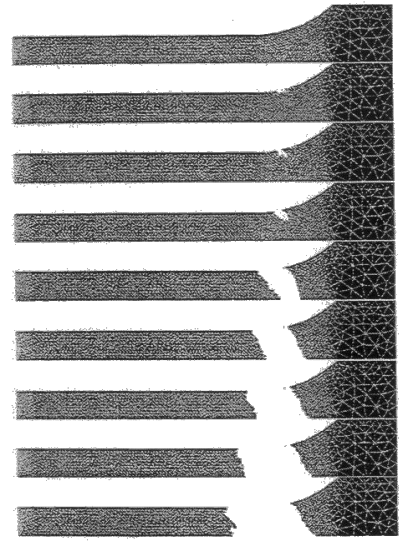

As an example of application (figure 2), numerical simulations [Pagano et al., 1999] on a tensile test showed a propagation of the phase transition initialized at the connection zone of the sample and spreading out over the gauge length in accordance with some recent experiments.

Figure 2 ; Evolution of phase transition during the loading

\subsubsection{The phase change: a thermomechanical coupling mechanism}

In a previous paragraph, we pointed out the properties of the quasiconvex energy $Q W$. This energy was deduced from an analysis at a microscopic scale. Another way to identify the mesoscopic potential is based on experiments on monocrystalline samples. The phenomenological features we saw led us to consider a convex energy at this scale. The confrontation between the micro and meso approaches and the phenomenological one was profitable. In this section, we present the retained mesoscopic free energy $W\left(T, \varepsilon, x_{k}\right)$ and the derived constitutive equations of the monocrystal, in order properly to describe different phenomena such as pseudoelasticity, reorientation effect and thermomechanical couplings accompanying the phase change.

The experiments [Balandraud, 2000] were carried out on a monocrystalline CuZnAl specimen. First, uniaxial load-unload tests were performed in the "low temperature region" (typically $22^{\circ} \mathrm{C}$ ). The apparent permanent strain observed in Figure 3 can be recovered by a heating-cooling process under zero stress. This effect, induced by martensite variant reorientation, leads us to consider, at low temperatures, a non-strictly convex property of the marginal energy :

$$
\underline{W}(T, \varepsilon)=\underset{x_{\mathrm{k}}}{\operatorname{Min}} W\left(T, \varepsilon, x_{\mathrm{k}}\right) \text {. }
$$

Note also that, once the reorientation strain is annealed, the stress, the strain and the temperature recover their initial values. Therefore, and from a thermomechanical point of view, we do not refer to plasticity to explain reorientation strain. 
Another interesting test concerns pseudoelasticity. If the load-unload is imposed in the "high temperature region" (typically $30^{\circ} \mathrm{C}$ ), we can observe (Figure 4a) a hysteresis loop in the strain-stress diagram that must be studied in the light of the thermodynamic framework presented in part 2. Moreover, temperature variations of the sample can be observed (Figure 4b) around the prescribed value (the temperature of the environmental chamber). These variations, though small, are induced by significant heat sources. Energy balance makes it possible to split the volume heat source $w_{\mathrm{ch}}^{\prime}$ into a part due to dissipative effects and another one due to thermomechanical coupling mechanisms. In all the tests performed, the first part was always small compared to the second $(<2 \%)$.

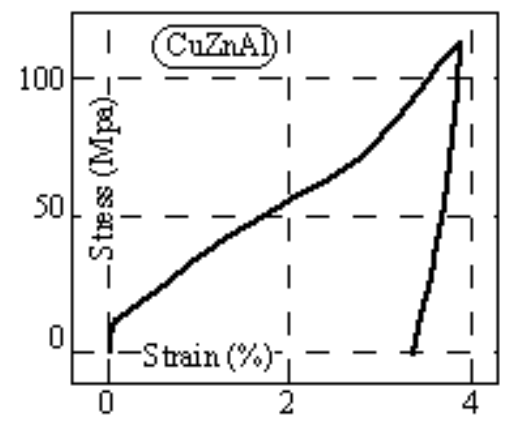

Figure 3 ; Stress-strain curve in a reorientation test

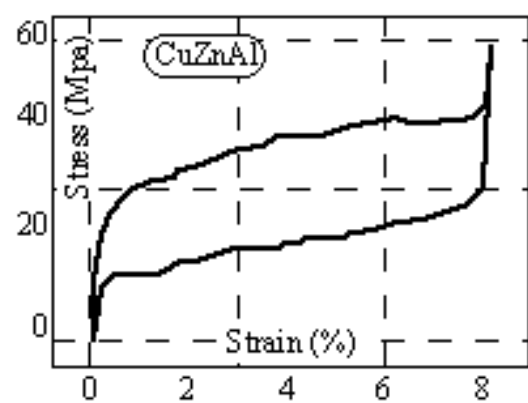

Figure $4 a$; Stress-strain curve in a pseudoelastic test

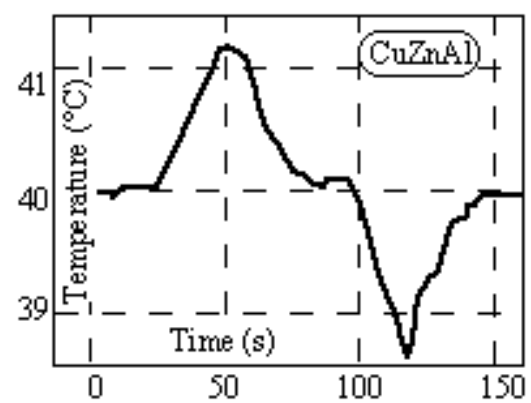

Figure $4 \boldsymbol{b}$; Temperature variation in a pseudoelastic test

Note that, for all uniaxial tests performed, we did not observe non-monotonous stressstrain curves. In all cases where non-monotonous load-displacement curves occurred, strain localization effects were seen (propagation of transformation band, [Wattrisse,1999, Balandraud, 2000]). All these remarks lead us to consider phase change in SMAs as an anisothermal process, and to use a quasiconvex energy density function. For the sake of simplicity and without any data on multiaxial behaviour, we chose a convex function to suitably describe the behaviour in terms of the kinetics of phase change. The retained free energy $\mathrm{W}$ is

$$
W(T, \varepsilon, x)=W^{\mathrm{el}}(T, \varepsilon, x)+W^{\mathrm{pc}}(T, \varepsilon, x)+I_{\mathrm{C}}(x) .
$$

Here $I_{C}$ is the characteristic function of admissible values of $x$, and 


$$
W^{\mathrm{el}}(T, \varepsilon, x)=\frac{1}{2} A\left(\varepsilon-\varepsilon^{\mathrm{pc}}(x)-\alpha T\right):\left(\varepsilon-\varepsilon^{\mathrm{pc}}(x)-\alpha T\right)-\frac{1}{2}\left(\frac{\rho C}{T_{0}}+\alpha A \alpha\right) T^{2},
$$

where the subscript pc stands for phase change, and $\varepsilon^{\mathrm{pc}}(x)=x_{1} \beta^{1}+x_{2} \beta^{2}+\ldots+x_{n} \beta^{n}$.

The values $\beta^{k}$ characterise the phase change strain associated with the variant $k$ and derive directly from microscopic studies of the crystallographic lattice.

$$
W^{\mathrm{pc}}=\frac{L}{T_{0}}\left(T-T_{\mathrm{A}}\right)\left(x_{1}+x_{2}+\ldots+x_{n}\right),
$$

with $L$ the latent heat and $T_{0}$ the reference temperature.

Now, in the formalism of standard materials, a choice of a dissipation potential $\phi$ leads to complementary laws, and the writing of intrinsic dissipation gives the expression for the transition domain:

$$
-A\left[\varepsilon-\alpha \theta-x_{k} \beta^{k}\right]: \beta^{k}+\rho \frac{L}{T_{0}}\left(T-T_{\mathrm{A}}\right) \in-\rho \partial_{\dot{x}_{k}} \phi-\rho \partial_{x_{k}} I_{C}, k=1, \ldots, n,
$$

where $\partial_{s} f$ stands for the partial sub-differential of $f$ with respect to $s$.

In the particular case of a zero dissipation potential $\phi$, the last expression reduces to

$$
-\sigma: \beta^{k}+\rho \frac{L}{T_{0}}\left(T-T_{\mathrm{A}}\right) \in-\rho \partial_{\mathrm{x}_{\mathrm{k}}} I_{\mathrm{C}}, k=1, \ldots n,
$$

and represents the transition domain in a temperature-stress plane $\left(T_{\mathrm{A}}\right.$ is the zero stress transition temperature). Figure 5 represents the numerical results obtained in a pseudoelastic test by using this model. The hysteresis loop is correctly predicted, and the temperature variations are consistent with experiment.

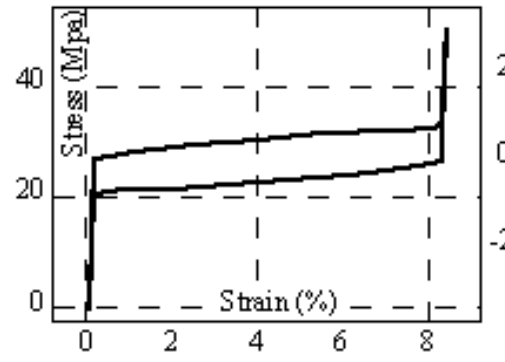

$\mathbf{a}$

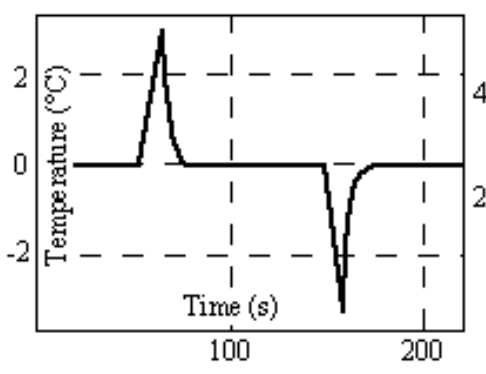

b

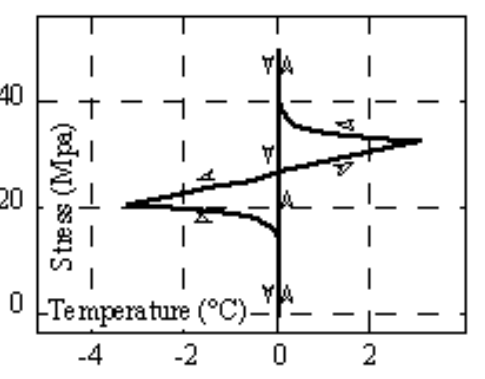

c

Figure 5; a) hysteresis due to thermomechanical couplings, b) temperature variations due to "stress-induced" phase change, c) thermodynamic path in the phase diagram corresponding to the load-unload anisothermal cycle.

Even if thermal effects due to dissipation are difficult to measure, it is legitimate to suppose that a slight irreversibility accompanies the phase change. To test the influence of slight dissipation, we chose

$$
\phi\left(\dot{x}_{1}, \dot{x}_{2}, \ldots, \dot{x}_{n}\right)=\frac{1}{2} K_{\mathrm{v}}\left(\dot{x}_{1}^{2}+\dot{x}_{2}^{2} \ldots+\dot{x}_{n}^{2}\right) \text {. }
$$


The existence of terms in $\dot{x}_{k}$ in the expression for the transition diagram expresses a dependence on the sense of transformation $(M \rightarrow A$ or $A \rightarrow M)$. Figure 6 shows that this slight dissipation has significant effects on the size of the hysteresis loop area, while the temperature evolutions with and without dissipation are hardly distinguishable.
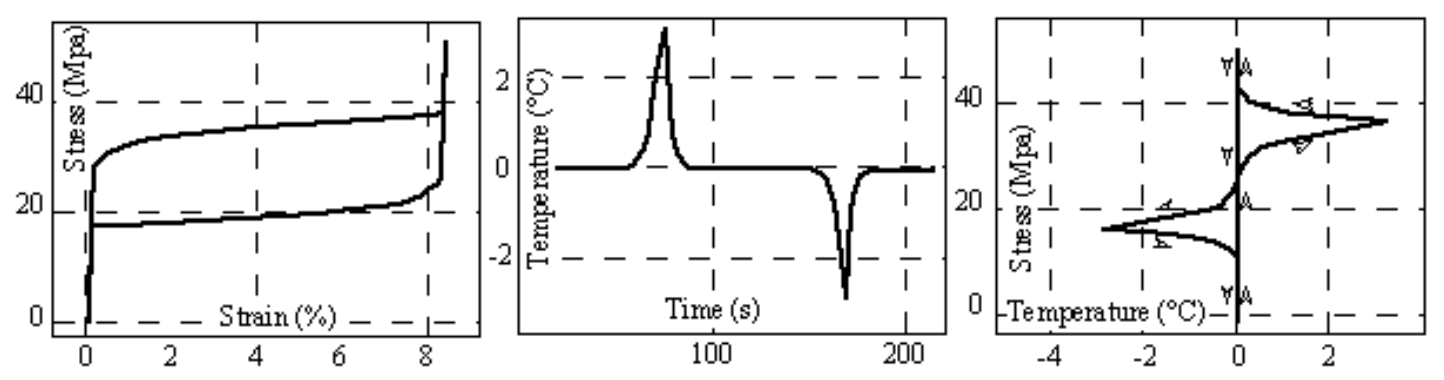

Figure 6; a) hysteresis due to thermomechanical couplings and to a slight intrinsic dissipation, b) temperature variations due to a "stress-induced" dissipative phase change, $\boldsymbol{c}$ ) corresponding path in the phase diagram.

These constitutive equations and the heat equation have been included in a finite element program to simulate the fully coupled thermomechanical behavior of monocrystalline SMAs [Peyroux, 1998, Balandraud, 2000]. It allows a correct prediction of pseudoelasticity, reorientation effect and recovery strain, it predicts phenomena such as asymmetry in tension-compression tests, time effects due to heat diffusion, inducing "relaxation".

\subsection{Macroscopic scale; towards modeling of the polycrystal behaviour}

\subsubsection{Macroscopic variables}

We now focus on the macroscopic behaviour of a SMA polycrystalline aggregate. We suppose that each grain behaves as a monocrystal, and the aim of this part is to derive the homogenized thermomechanical characteristics of the material.

The first assumption is that the macroscopic behavior can be derived from the thermomechanical response of a representative volume element (RVE). The observation of a polycrystalline sample by means of electronic and optical microscopy, allowed us to gather data on the crystallographic texture, the shape and the statistical representation of the grains in the RVE. (Figure 7)

In the following, we will consider that all the grains differ only by their crystallographic orientation.

It is established [Suquet, 1984] that for microscopic constitutive equations containing internal variables, "the homogenized law does not reduce to a single equation" on the macroscopic domain. "The knowledge of the macroscopic law requires as data the 
(mesoscopic) state variables". An alternative and pragmatic attitude is to link the macroscopic thermomechanical variables together, by prescribing thermomechanical loading and numerically solving the problems on the RVE. The RVE is considered as a virtual sample, and the finite element code as a virtual thermomechanical testing device.

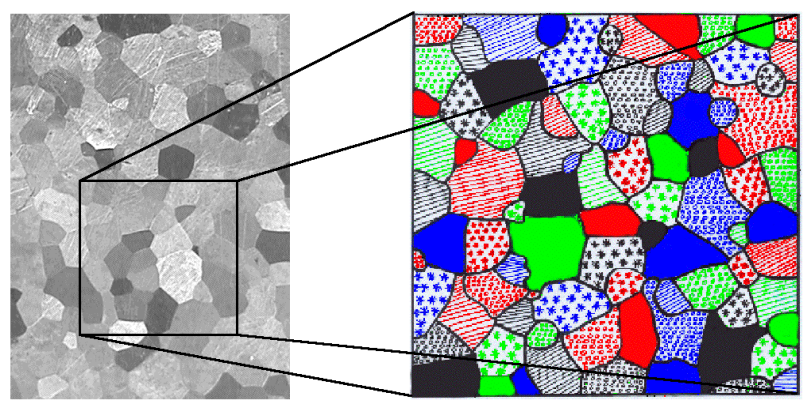

Figure 7; RVE of a polycrystalline sample of CuZnAl SMA obtained by image analysis; Different colors stand for different crystallographic orientations

The set of variables used at the mesoscopic level is $\left(T, \varepsilon, x_{k}\right)$, and additional variables such as the stress tensor $\sigma$ or the heat influx vector $q$, can be deduced from the initial set owing to the transition diagram or the heat equation.

On the macroscopic level, we consider the classic and natural macroscopic strain and stress tensors $E$ and $\Sigma$ defined as average values of $\varepsilon$ and $\sigma$.

$$
E=<\varepsilon>\text { and } \Sigma=<\sigma>\text {. }
$$

At first we assume a quasi-homogeneous mesoscopic temperature field, the value of which is identified with the macroscopic temperature. Recall that the quasihomogeneity of the temperature field does not imply the homogeneity of its gradient.

At this point $E^{\mathrm{pc}}$, part of macroscopic strain due to phase change, is given by $E^{\mathrm{pc}}=E-A^{-1} \Sigma-\alpha\left(T-T_{0}\right)$.

It remains to define $X$ : macroscopic equivalence to the volume proportion of phase change. Deriving $\bar{L}$, macroscopic latent heat, from $\bar{\rho} \bar{L}=\langle\rho L\rangle$, we can propose as a definition of $X: \bar{\rho} X=\langle\rho L x\rangle$. This macroscopic proportion has to be regarded as an energy indicator of the advancement of phase change. The value of $X$ is 0 if the polycrystal is completely austenitic, and 1 if completely martensitic. On the other hand a value between 0 and 1 can be reached under several microscopic configurations.

\subsubsection{Some numerical results on the macroscopic behavior of a polycrystalline SMA}

A convenient set of macroscopic variables being established, we present in this part some results of finite element simulations on the RVE.

First, we performed a so-called pseudoelasticity test consisting of a load-unload path at a room temperature upper than $T_{\mathrm{A}}$ and the different results are plotted in Figure 8. The imposed strain is increased from point $a$ to point $d$, and maintained at a certain level 
between points $b$ and $c$. In the particular stage $b c$, we can observe the role played by the thermomechanical couplings. The material keeps on transforming ( $X$ increases), the stress relaxes while the temperature returns to the imposed value. Now, concerning the entire test, the evolution of the different variables is consistent with experimental results obtained on polycrystalline SMA. This macroscopic behaviour is of course different to the monocrystalline one and corresponds to the particular ordering of grains chosen in the RVE.
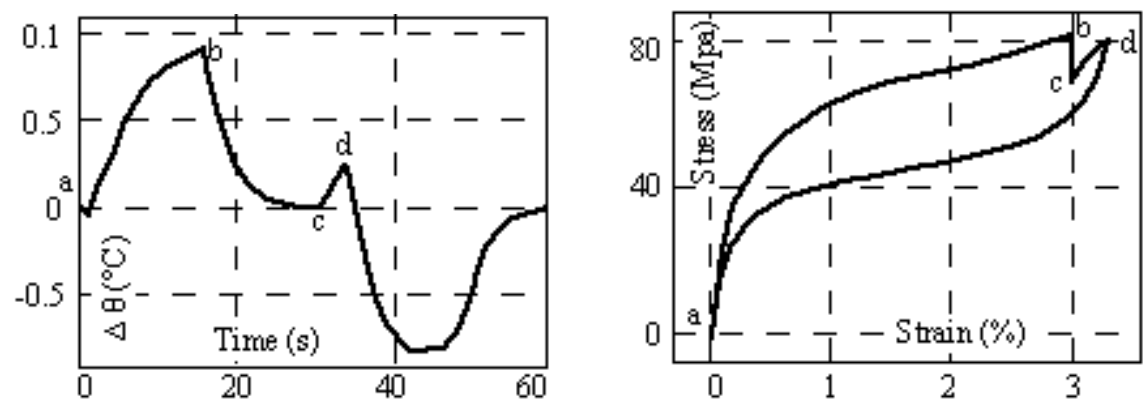

Figure 8; Pseudoelastic “numerical” test

From Figures 7 we can deduce some interesting points in the macroscopic transition domain. If we perform this analysis at several room temperatures, we obtain isovalues of transformation in the stress-temperature plane (Figure 9). An interesting result is that, according to the slope of the isovalues, it appears to be difficult to reach a complete transformation under reasonable stresses. In fact, some grains in the RVE have a noncompatible crystallographic orientation with respect to the imposed stress, while others transform easier. Note that taking into account a non zero dissipation leads to "moving lines" with regard to the direction of transformation.

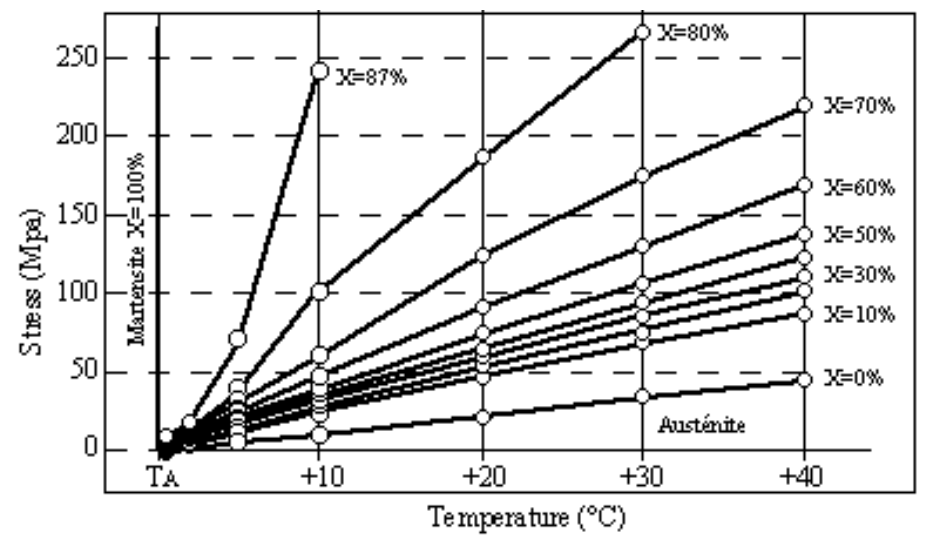

Figure 9 ; Phase diagram for uniaxial tension test performed; $T \geq T_{A}$ and $d_{l}=0$.

The possibilities of this approach have been widely developed in [Balandraud, 2000]. For instance, low-temperature tests allowed determining the value of the macroscopic recoverable strain due to variants reorientation at zero-stress. This numerical tool could also be used to investigate multiaxial behavior of polycrystalline SMAs and to study the influence of various dissipation potentials. Finally, this approach giving access to 
mesoscopic behaviour, the evolution of phase change in each grain might be soon correlated with optical observations to check the consistency of this multiscale modeling.

\section{CONCLUDING COMMENTS}

We have tried to present the different points of view developed at the LMGC concerning the understanding of the SMA behaviour. This variety of approaches comes from the different analysis methods and from the chosen scales of description. Even though certain theoretical concepts were borrowed from other works in the literature, all our approaches led to original numerical tools. From an experimental point of view, the LMGC has developed for more than ten years infrared image processing techniques allowing us to perform calorimetric balances. For of SMAs, these tools made it possible to observe the strong thermomechanical coupling that the martensite transformation represents. At meso and macroscopic scales, the experiments highlighted the quasistatic and slightly dissipative character of the kinetics of this solid-solid phase transition.

\section{REFERENCES}

[Abeyaratne et al., 1993] Abeyaratne, R, Knowles, J. K., A continuum model of thermoelastic solid capable of undergoing phase transitions, J. Mech. Phys. Solids, Vol.41, pp. 541-571.

[Auchmuty, 1989] Auchmuty, G.; Duality algorithms for non convex variational principles; In: Numerical Functional Analysis and Optimization, Vol. 10, pp 211-264.

[Balandraud, 2000] Balandraud, X., Changement de phase et changements d'echelle dans les alliages à mémoire de forme, Thesis of University Montpellier II.

[Ball et al., 1987] Ball, J.M., James, R., Fine phase mixtures as minimizers of energy, Arch. Ration. Analysis, 100, pp. 15-52.

[Ball et al., 1992] Ball, J.M., James, R., Proposed experimental tests of a theory of fine microstructure and the two-well problem, Phil. Trans. R. Soc. London, A, 338, pp; 389-450.

[Bhattacharya et al., 1997], Battacharya, K., Kohn, R., Elastic energy minimization and the recoverable strains of polycrystalline shape-memory materials, Arch. Rat. Mech. and Ana., pp.99-181.

[Chrysochoos et al, 1993], Chrysochoos, A., Pham, H., Maisonneuve, O., An experimental analysis of a thermomechanical behaviour of a CuZnAl shape memory alloy, C.R. Acad. Sci, Paris, t.316, Série II, pp.1031-1036.

[Chrysochoos et al, 1995], Chrysochoos, A., Löbel, M., Maisonneuve, O., Thermomechanical coupling of pseudoelastic behaviour of CuZnAl and NiTi alloys, C.R. Acad. Sci, Paris, t.320, Série IIb, pp.217-223.

[Chrysochoos et al., 2000], Chrysochoos, A., Louche, H., An infrared image processing to analyse calorific effects accompanying strain localisation, to appear in : Int. J. Engng. Sci..

[Collins, 1993], Collins, C., Computation of twinning, microstructure and phase transition, IMA, vol54.

[Dacorogna, 1982], Dacorogna, B., Quasiconvexity and relaxation of nonconvex problems in the calculus of variation. Journal of functional analysis, Vol. 46, pp.102-118.

[Ericksen, 1984], Ericksen, J.L., The Cauchy-Born hypotheses for crystals. Phase Transformations and Material Instabilities in Solids, Ed. M. Gurtin, Academic Press, Vol. 46, pp.61-78.

[Frémond, 1987], Frémond, M.; Adhérence des solides; Journal de Mécanique Théorique et Appliquée, Vol 6, pp 383-407. 
[Frémond, 1987], Frémond, M.; Matériaux à mémoire de forme; C. R. Acad. Sci. Paris, ", Vol 304, Série 2, pp 239-244.

[Germain, 1973], Germain, P, Cours de Mécanique des Milieux Continus, Masson et Cie Ed, p.417.

[Germain et al., 1983], Germain, P, Nguyen, Q. S., Suquet, P., Continuum thermodynamics, J. of Applied Mechanics, vol. 50, pp. 1010-1020.

[Glansdorff et al., 1971], Glansdorff, P., Prigogine, I., Structure, Stabilités, Fluctuations, Masson et Cie Ed., p.288.

[James, 1987], James, R.D.; The stability and metastability of quartz, Metastability and Incompletely Posed Problems, Springer-Verlag, IMA Vol 3, pp 147-176.

[James et al., 1989], James, R., Kinderlehrer, D., Theory of diffusionless phase transformations, Lecture Notes in Physics, 344, pp. 51-84.

[James et al., 1993], Biaxial loading experiments on CuAlNi single crystals., AMD-Vol 181, Experiments in Smart Materials and Structures, ASME.

[Kohn, 1991], Kohn,R.V., The relaxation of a double-well energy, Continuum Thermodyn., 3, pp. 193.

[Kondepudi et al., 1998], Kondepudi, D, Prigogine, I., Modern Thermodynamics, Wiley \& Sons Ed., p.486.

[Leclercq et al., 1996], Leclercq, S.; Lexcellent, C.; A general macroscopic description of the thermodynamical behavior of shape memory alloys; J. Mech. Phys. Solids, Vol. 44, pp.953-980.

[Licht, 1997], Licht, C., Numerical experiments about non-convex bulk energy functions, proceedings of Engineering Mechanics Today 97, Hanoi Vietnam, 1997.

[Licht, 2000], Licht, C., Interaction energy in the macroscopic modelling of phase transition in solids, Euromech 2000, Metz.

[Müller et al., 1991], Müller, I, Xu, H, On pseudoelastic hysteresis, Acta Met. Mater., 39, 263,

[Pagano et al., 1998], Pagano, S.; Alart, P.; Maisonneuve O.; Solid-solid phase transition modeling. Local and global minimizations of non-convex and relaxed potentials. Isothermal case for shape memory alloys; Int. J. of Eng. Sc. 36, pp. 1143-172.

[Pagano et al., 1999], Pagano, S.; Alart, P.; Solid-solid phase transition modeling: relaxation procedures, configurational energies and thermomechanical behaviors; Int. J. of Eng. Sc. to appear.

[Papon et al., 1999], Papon, P., Leblond, J., Meijer, Paul H.E., Physique des Transitions de Phases, Dunod Ed., p.390.

[Patoor et al., 1994], Patoor, E., Berveiller, M, Technologie des AMF, Hermes ed., p. 286.

[Peyroux et al., 1998], Peyroux, R., Chrysochoos, A., Licht, C., Löbel, M., Thermo-mechanical couplings and pseudoelasticity of shape memory alloys, I. J. Eng. Sci., Vol. 36, N4, pp. 489-509.

[Raniecki et al., 1992], Raniecki, B.; Lexcellent, C.; Tanaka, K.; Thermodynamic models of pseudoelastic behavior of shape memory allows; Arch. Mech. Vol; 44, pp. 261-284.

[Stavroulakis et al., 1993], Stavroulakis, G.E.; Panagiotopoulos,P.D.; Convex multilevel decomposition algorithms for non-monotone problems; Int. J. for Num. Meth. in Eng., Vol. 36, pp. 1945-1966.

[Suquet, 1984], Suquet, P.M.; Approach by homogenization of some linear and nonlinear problems in solids mechanics; Ed. J.P. Boelher, Colloques Internationaux du CNRS, n³19, pp.77-117.

[Wattrisse, 1999], Wattrisse, B., Etude cinématique de la localisation dans des aciers par intercorrélation d'images, Thesis, University of Montpellier II.

[Zhong et al., 1996], Zhong, S.G.; Batra, R.C.; Modeling of macroscopic response of phase transformation materials under quasi-static loading ; J. Elasticity 44, pp. 145-160. 